\title{
Bereavement in Difficult Times
}

Vita Poštuvan

Assoc. prof. Vita Poštuvan works as a researcher and Deputy Head of the Slovene Centre for Suicide Research (UPIAM) and Department of Psychology (FAMNIT) at the University of Primorska. She leads the work related to clinical-research and public-health interventions in suicidology, bereavement, crisis interventions and psychotherapy. She has participated in major European and Slovenian studies in the field of (public) mental health, suicidology and psychology, which were published in international journals. She has worked at the National Center of Neurology and Psychiatry (NCNP) in Tokyo, Japan as well as in the Suicide Research Group, Department for Psychoanalysis and Psychotherapy, Medical University of Vienna, Austria, as a fellow researcher. Her professional work is also focused on psychological counselling, psychotherapy and prevention. As a psychological counsellor and cognitive-behavioural therapist she offers psychological support for students and individuals and implements mindfulness-based cognitive therapy. She is a member of national unit for psychological research within the civil protection.

Abstract. Death and suicide are inseparable from grief. As we know, bereavement is a process of coming to terms with the loss of a loved one, full of difficult emotional challenges. One of the most important elements in this process are the support of people in the social network, community, and systems. These are usually expressed through rituals around death. In suicide cases, bereaved people often feel that there is less community support because of the stigma surrounding the cause of death. Bereaved people feel that those around them express their condolences with 'empty words,' distance, or fear that the bereaved will blurt out their emotions. Therefore, avoidance and a lack of authentic support is a common experience of bereaved people after a suicide. In the time of the Covid-19 pandemic, this is even more evident. Many systems, such as schools or places of employment, which often provided a natural way to connect with others and give the bereaved opportunity for meaningful interaction, were closed for extended periods of time. This has implications for complications in the grieving process, such as prolonged grief disorder (PGD) or complicated grief (CG). 